\title{
Recomendações de recrutamento em ecossistemas de startups: uma revisão sistemática da literatura
}

\author{
Leonardo Herdy Marinho ${ }^{1}$, Mônica Ferreira da Silva ${ }^{1}$, Jonice de Oliveira \\ Sampaio ${ }^{1}$ \\ ${ }^{1}$ Programa de Pós-graduação em informática (PPGI) - Universidade Federal do Rio de \\ Janeiro (UFRJ) - Rio de Janeiro - RJ - Brasil
}

leonardomarinho@ufrj.br, mfsilvamailegmail.com, jonice@dcc.ufrj.br

\begin{abstract}
Finding professionals who meet the constant openings in startups is a great challenge considering that their disruptive model requires people with increasingly specific knowledge. To find people who meet the requirements, recruiters are increasingly using techniques, templates and tools to support the hiring decision. To recommend hires, you first need to understand the state-of-the-art of research in the area of recruiting recommendations in startup ecosystems. Therefore, we carried out a systematic literature review in which 854 articles were analyzed, which provided us with data on the scientific progress of the investigated area and research focuses.
\end{abstract}

Resumo. Encontrar profissionais que atendam às constantes aberturas de vaga em startups é um grande desafio tendo em vista que o modelo disruptivo das mesmas exige pessoas com conhecimentos cada vez mais especificos. Para encontrar pessoas que atendam aos requisitos, recrutadores utilizam cada vez mais técnicas, modelos e ferramentas para apoiar a decisão de contratação. Para recomendar contratações, é necessário primeiro entender o estado da arte da pesquisa na área de recomendações de recrutamento em ecossistemas de startups. Por isso, realizamos uma revisão sistemática da literatura em que 854 artigos foram analisados, o que nos forneceu dados sobre o progresso científico da área investigada e focos de pesquisa.

\section{Introdução}

Segundo [Soetanto e Jack 2013], a pluralidade de indivíduos envolvidos em determinado ambiente pode influenciar de forma positiva no desenvolvimento de negócios e aumentar a possibilidade de parcerias bem sucedidas. Entretanto, identificar parceiros adequados para projetos vai se tornando mais difícil com o crescimento da rede de indivíduos, pois é necessário conjugar os interesses mútuos [Ikenami 2016]. Neste contexto, é importante facilitar o recrutamento e seleção de profissionais por meio de recomendações baseadas nos interesses dessas redes de indivíduos que são tipicamente complexas. Encontrar especialistas para um domínio não seja por si só trivial [Birgit Pröll and Hannes Werthner 2005]. Antes de realizar esta pesquisa, notamos que não existiam revisões de literatura detalhadas abordando o tema de recomendações de recrutamento utilizando análise de redes sociais. Para suprir tal demanda foi necessário conduzirmos uma revisão sistemática de literatura (RSL) que segundo [Kitchenham 2004] é uma das formas 
existentes de identificar, interpretar e avaliar todos os trabalhos de pesquisa disponíveis e relevantes. Uma das principais razões para se realizar revisão sistemática é que esta resume as evidências existentes correlatas a um determinado campo de estudo ou tecnologia. Este trabalho tem o intuito de visualizar e categorizar de as publicações realizadas na temática de sistemas de recomendações de recrutamento com base em análise de redes sociais. Para a revisão, optamos pela técnica de busca automatizada. $\mathrm{O}$ objetivo desta RSL é compreender os conceitos da criação de novos relacionamentos entre os indivíduos no meio profissional, métodos de recomendação aplicados por recrutadores e atuais dificuldades reportadas na busca por profissionais através de recomendações. Através da RSL foi possível condensar o atual estado da arte dos estudos desses tópicos em conjunto. É válido ressaltar que este estudo é parte de um trabalho em andamento onde os autores investigam o uso de sistemas de recomendação e análise de redes sociais para a tomada de decisão no âmbito de recrutamento de novos talentos para suprirem vagas de trabalho no meio tecnológico com base no fenômeno da cadeia de oportunidades [White, 2013] e descobrir futuramente como auxiliar recomendações de recrutamento em ecossistemas de startups.

\section{O processo da revisão sistemática de literatura}

Esta RSL foi baseada no processo descrito por [Kitchenham 2004], segundo o qual existe uma série de etapas bem definidas para a correta execução de uma RSL. A etapa inicial é a definição do protocolo de pesquisa. Kitchenham aborda o protocolo como a parte do trabalho que especifica as questões de pesquisa, estratégias que serão utilizadas para condução da revisão, critérios de inclusão e exclusão, extração e síntese dos dados.

\subsection{Questões de pesquisa}

Considerando que as questões de pesquisa devem exemplificar os objetivos do estudo de RSL, as seguintes questões foram elaboradas:

QP1: Quando e onde os estudos têm sido publicados? QP2: Do ponto de vista de recomendações, quais aspectos têm sido focados? QP3: Quais problemas têm sido apontados com a necessidade de novos estudos? QP4: Qual contexto está sendo estudado? QP5: Quais são os tipos de contribuição encontrados? QP6: Quais características precisam ser levadas em conta para eleger um profissional? QP7: Como encontrar um profissional que tenha a melhor adequação ao trabalho? QP8: Que métodos científicos de pesquisa têm sido usados?

\subsection{Fontes de pesquisa utilizadas}

Os trabalhos consultados para a condução desta pesquisa foram buscados através dos seguintes repositórios acadêmicos: Scopus, Springer Link, IEEE Digital Library, ACM Digital Library, Science Direct, Sol (SBC) e Scielo. O uso de pelo menos dois indexadores e duas bibliotecas combinadas é suficiente para que os resultados sejam satisfatórios e não enviesados por virem de uma única fonte de trabalhos científicos [Tore Dyba, et al. 2007]. Estas bases foram escolhidas cuidadosamente buscando abraçar a área mãe desta pesquisa que é Ciência da Computação e ao mesmo tempo ter uma boa interseção com outras áreas como negócios e gestão de recursos humanos. 


\section{Termos usados na pesquisa (palavras-chave)}

Os termos que foram utilizados nesta RSL foram agrupados em uma lista, e, quando combinados formaram a string de busca. Os termos estão em língua inglesa, por este ser o idioma amplamente utilizado por pesquisadores e motores de busca. É válido citar que estes termos foram encontrados com os estudos realizados na pesquisa bibliográfica preliminar, pesquisa tal que consistiu em uma revisão menos formal realizada no Google Scholar e com artigos obtidos após reuniões com pesquisadores referência nas áreas pesquisadas. Tendo conhecimento das keywords mais utilizadas na área, foi possível definir quais palavras seriam mais assertivas para a definição da string.

- professional, talent, employed.

- job matching, job fit, job hunting, job seeking.

- recommendation, recruitment, selection, team formation.

- social network, social network analysis

Para as buscas realizadas nas bases da Scielo e Sol SBC os termos foram traduzidos para a língua portuguesa de forma experimental visando observar se haviam estudos com o tema investigado para consultarmos.

- profissional, talento, empregado.

- correspondência de trabalho, procura por emprego, adequação ao trabalho.

- recomendação, recrutamento, seleção, formação de time.

- rede social, análise de rede social.

\section{String de busca}

Várias tentativas foram realizadas combinando termos chave e elementos importantes dentro da temática observada até que a string retornasse resultados satisfatórios. Para este trabalho, realizamos 12 testes combinando as palavras chave levantadas na pesquisa bibliográfica preliminar. A string de busca aceita como satisfatória foi a que melhor equilibrou a quantidade de trabalhos para serem analisados dentro do tempo reservado para a RSL e da pesquisa de mestrado que a exigiu. Foi realizada uma rigorosa leitura dos títulos e resumos de forma amostral para entender se os resultados retornados pela base de busca eram suficientes a cada teste. A base utilizada para foi a Scopus, por esta ser amplamente recomendada e utilizada em trabalhos de ciência da computação. Aqui, demonstramos uma string de busca utilizada e disponibilizamos um endereço ${ }^{1}$ que detalha as demais. Não inserimos a palavra startup na string para não diminuir o espaço amostral retornado. Scopus: TITLE-ABS-KEY ((recruitment OR selection OR job matching OR job seeking OR job hunting OR job fit OR team formation) AND recommendation AND (talent OR employed OR professional)) AND social network AND (LIMIT-TO (SUBJAREA, "COMP") OR LIMIT-TO (SUBJAREA , "BUSI"))

\footnotetext{
${ }^{1}$ Strings de busca:

https://github.com/Leomhl/masters degree archives/blob/master/rsl/strings busca.md
} 


\section{Processo de seleção preliminar}

Após a busca por trabalhos nas sete bases citadas anteriormente, foram selecionados os artigos que apresentaram informações no título e no resumo relacionado às questões de pesquisa. Os trabalhos que tiverem as palavras chave (keywords) definidas na string de busca, mas não eram relacionados ao tema descartamos, considerando "falso positivo".

\section{Critérios de inclusão e exclusão}

[Kitchenham 2004] diz que devem ser seguidos critérios de inclusão e exclusão para os artigos que são retornados pela busca nas bases. Para tal, foram definidos os critérios e seguidos de forma rigorosa para selecionar ou eliminar os estudos obtidos. Sendo eles, critérios de inclusão: CI1: Abordar o tema de recomendação em recrutamento e/ou entrevistas de emprego; CI2: Estar relacionado à análise de redes sociais para ecossistemas empreendedores; CI3: Ser da área de ciência da computação ou Negócios/Gestão/Recursos humanos;

Os critérios de exclusão utilizados foram: CE1: Não estar disponível para leitura; CE2: O estudo não possui um resumo; CE3: Não se encaixa nos critérios de inclusão;

\section{Critérios de qualidade}

Em RSL, após a seleção final dos estudos obtida pela aplicação dos critérios de inclusão e exclusão e considerando o texto completo, uma avaliação de qualidade mais detalhada precisa ser feita [Kitchenham 2004]. Por isso, é importante encontrar critérios de qualidade que possam ser aplicados de maneira similar ao longo dos diferentes tipos de trabalhos identificados [Claes Wohlin, et al. 2012]. Cada ganhou peso 1 caso obtivesse como resposta "sim", e, os trabalhos que obtiverem nota igual ou superior a 2 foram selecionados para a próxima etapa. Abaixo, as questões que utilizamos:

QQ1: Há informações suficientes sobre a coleta e análise de dados para verificar a pesquisa? QQ2: A abordagem metodológica é bem definida e bem descrita? QQ3: O artigo apresenta uma descrição completa da solução aplicada na abordagem? QQ4: Este artigo é relevante para a pesquisa?

\section{Análise dos resultados}

Após a aplicação da string de busca nas bases já citadas, obtemos o retorno está na Tabela 1. Destacar que esta busca de artigos foi realizada entre os meses de abril e maio de 2020 e rodada novamente no começo de 2021 para buscar novos trabalhos na área.

\begin{tabular}{|l|c|l|c|}
\hline \multicolumn{1}{|c|}{ Base científica } & Resultados & \multicolumn{1}{c|}{ Base científica } & Resultados \\
\hline Scopus & 229 & Science Direct & 13 \\
\hline IEEE Digital Library & 116 & Scielo & 4 \\
\hline Springer Link & 463 & Sol SBC & 0 \\
\hline ACM Digital Library & 29 & Total & $\mathbf{8 5 4}$ \\
\hline
\end{tabular}

Tabela 1. Resultados da busca por estudos 


\section{Etapas de filtragem dos trabalhos}

Cada etapa foi utilizada como um funil que selecionou os artigos que atenderam ao protocolo desta RSL. Etapa 0, remoção de todos os estudos duplicados. Etapa 1, seleção com base na leitura do título, resumo e palavras chave e critérios de inclusão e exclusão. Etapa 2, leitura completa dos estudos, corrigindo a conformidade dos trabalhos de acordo com os critérios de inclusão e exclusão. Ao ler todo o trabalho foi possível notar o quão próximo ou distante eles estavam dos critérios, então, corrigimos o critério aplicado anteriormente com a leitura mais superficial realizada na Etapa 1. Critérios de qualidade, ao aplicar os critérios de qualidade e selecionando os estudos com nota igual ou maior a 2. Após a aplicação das etapas, foi realizada a extração de dados. Para tais etapas, pontuaremos os resultados obtidos para cada pergunta e seus respectivos detalhes. A Tabela 2 detalha os dados de cada etapa e número total de artigos que passaram por cada uma delas.

\begin{tabular}{|c|c|c|}
\hline Etapa & Entrada & Saída \\
\hline Etapa 0 & 850 & 783 \\
\hline Etapa 1 & 783 & 65 \\
\hline Etapa 2 & 65 & 44 \\
\hline Qualidade & 44 & 33 \\
\hline Total & - & $\mathbf{3 3}$ \\
\hline
\end{tabular}

Tabela 2. Resultado da aplicação de etapas

\section{Resposta para as questões de pesquisa}

QP1 - Quando e onde os estudos têm sido publicados? O intuito desta questão foi realizar uma parte de descoberta bibliométrica para entender quais são os países que mais investiram no tema de recomendações de recrutamento e formação de times e quando foi feito. As Figuras 1 e 2 trazem detalhes dos resultados.

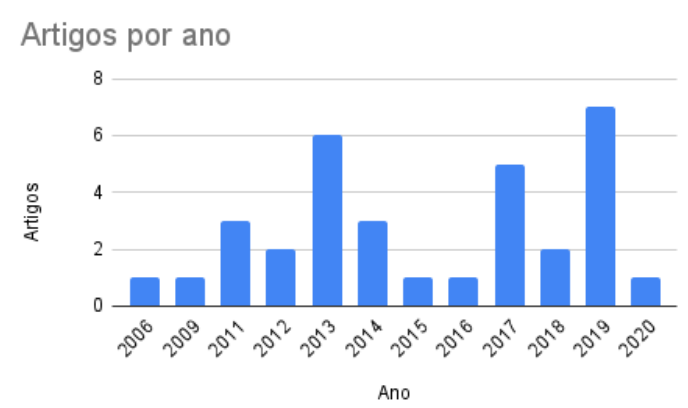

Figura 1. Artigos por ano

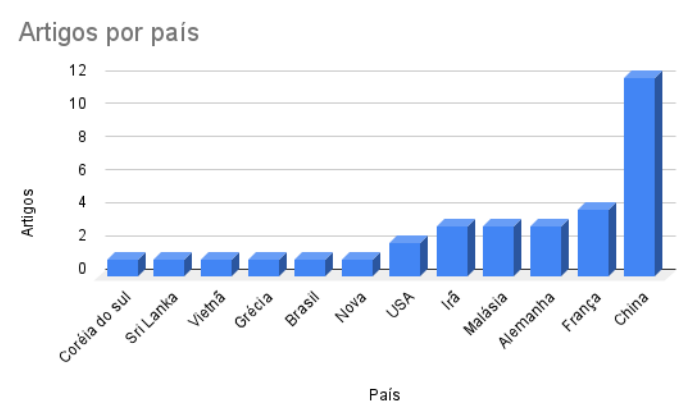

Figura 2. Artigos por país

QP2 - Do ponto de vista de recomendações, quais aspectos têm sido focados? Para detalhar as maiores áreas de investigação vigentes, criamos categorias para classificar cada estudo de maneira a gerar uma visão geral do que está sendo pesquisado. 
Categoria 1 (11): Pesquisa que provê uma forma para candidatos/estudantes encontrarem vagas por meio de recomendações que combinam dados curriculares e exigências das vagas. Categoria 2 (9): Pesquisa que provê uma forma de os recrutadores encontrarem candidatos através de recomendações usando dados de redes sociais, plataformas de recrutamento ou currículo. Categoria 3 (3): Pesquisa que provê otimização de classificadores, modelos, representações visuais ou algoritmos que trabalhem com a recomendação de usuários, vagas, times e afins. Categoria 4 (5): Pesquisa que provê formas receberem recomendações de candidatos que se complementam para a construção de times. Categoria 5 (2): Pesquisa que provê uma recomendação de carreira com base no currículo de um aluno ou profissional. Categoria 6 (2): Pesquisas sobre como recomendar perguntas de entrevista aos recrutadores. As pesquisas desta categoria visam recomendar perguntas e questionários para o ato de entrevistar um candidato à vaga. Categoria 7 (1): Recomendação de itens não preenchidos no currículo de candidatos com comparação de perfil entre o candidato e pessoas similares. Categoria 8 (1): Extração de dados de recrutamento de plataformas de anúncio de vagas e redes sociais. A soma gera 34, um artigo entrou em 2 categorias.

QP3 - Quais problemas têm sido apontados com a necessidade de novos estudos? Detalhamos uma lista dos principais trabalhos em aberto que foram encontrados: 1 Estudar a classificação incorreta de indivíduos levando em conta informações não verdadeiras em seus currículos. 2 - Investigar o efeito da combinação de similaridade de classificação com outras métricas de similaridade social. 3 - Estudar a satisfação do contratante, mantendo os contratados e as expectativas, uma vez que a recomendação de emprego é recíproca. 4 - Analisar possibilidades de o usuário escolher um modelo de seleção de times adequado no estágio de partida a frio e investigar a possibilidade de aplicar essa abordagem a outros domínios. 5 - Além do tempo e da disponibilidade de recursos, existem outros fatores que afetam a recomendação dinâmica no sistema de recrutamento. $\mathrm{O}$ contexto formado na alta e baixa disponibilidade de vagas influencia $\mathrm{o}$ desejo do candidato ao trabalho. Adicionar esses fatores a modelos de recomendação.

QP4 - Qual contexto está sendo estudado (academia, indústria, governo, projeto ou organização)? Ao analisar o contexto em que os trabalhos obtidos foram aplicados foi observado que há uma forte concentração de estudos focados para o ambiente chamado de "indústria". No total, foram 26 trabalhos enquadrados no contexto da indústria e 5 no da academia. Isso nos mostra que tais pesquisas são mais focadas no meio privado.

QP5 - Quais são os tipos de contribuição encontrados (método, processo, modelo, métrica, ferramenta...)? A Tabela 3 trás os resultados obtidos na leitura dos 33 trabalhos.

\begin{tabular}{|l|c|c|c|}
\hline \multicolumn{1}{|c|}{ Tipo } & Quantidade & Tipo & Quantidade \\
\hline Modelo & 10 & Método & 4 \\
\hline Framework & 10 & Não se aplica & 2 \\
\hline Ferramenta & 7 & - & - \\
\hline
\end{tabular}

Tabela 3. Tipos de contribuições encontrados na RSL

QP6 - Quais características precisam ser levadas em conta para eleger um profissional? Saber quais características são as mais importantes a serem para selecionar corretamente 
um bom profissional para uma vaga é fundamental para o sucesso da escolha. Dos 33 estudos foi possível extrair esta resposta de 20. A Tabela 4 detalha os dados obtidos.

\begin{tabular}{|l|l|l|l|}
\hline Característica & Aparições & Característica & Aparições \\
\hline Habilidades técnicas & 15 & Multidisciplinaridade & 2 \\
\hline Experiência profissional & 12 & Áreas de interesse & 2 \\
\hline Grau de escolaridade & 8 & Lealdade a um emprego & 2 \\
\hline Informações demográficas & 3 & Hobbies & 1 \\
\hline
\end{tabular}

Tabela 4. Características a observar para eleger um bom profissional

QP7 - Como encontrar um profissional que tenha a melhor adequação ao trabalho? Dos 33 trabalhos, foi possível extrair esta informação de 11. A seguir, listamos as respostas obtidas nos estudos observados: 1 - Através de filtragem colaborativa comparando o conteúdo textual das vagas com o conteúdo do currículo do aluno e combinando com qual trabalho pessoas de perfis similares têm. 2 - Deixando que o candidato escolha qual tipo de trabalho ele prefere. $\mathbf{3}$ - Cruzando as habilidades do candidato com as exigências da vaga. 4 - Analisando as capacidades técnicas e habilidades e considerar a coautoria dele em participação com outros membros da equipe em projetos e pesquisas acadêmicas. 5 - Selecionar as habilidades do candidato e categorizar em quais áreas faz mais sentido ele estar. Ex.: Alto conhecimento em SQL e infraestrutura de servidores arremete o profissional para a área de infraestrutura de bancos de dados. 6 - Utilizando recomendação por filtragem híbrida entre perfil do candidato e vaga, considerando variáveis de adequação do perfil ao que os empregadores esperam do candidato. 7 - Com base em suas habilidades (skills) realizar perguntas mais assertivas escolhidas computacionalmente para que na entrevista seja possível mapear melhor o conhecimento e personalidade do candidato. 8 - Combinando as exigências reportadas por recrutadores com o perfil dos candidatos e descobrindo o quão próximo um candidato é das expectativas que a equipe tem para o novo integrante. 9 - Buscando um profissional o mais próximo possível das competências exigidas pela vaga de emprego, mesmo que faltem algumas características em específico ou que "sobre" algum elemento que a vaga não exija, mas que o candidato tenha. 10 - Calculando a similaridade entre campos chave (Título, Experiência, Formação e Interesses) e o teor das vagas. 11 - Utilizando algoritmos de similaridade como o SVM em vetores de habilidades.

QP8 - Que métodos científicos de pesquisa têm sido usados? Nesta pergunta buscamos entender quais tipos de contribuições são mais presente no meio das pesquisas da área. Para tal, dos 33 estudos descobrimos que 24 eram experimentação, 8 estudos de caso e 1 não foi possível obter esta informação.

\section{Validade}

Para garantir a validade e o não viés na condução do RSL, todo o processo de criação e validação do protocolo foi acompanhado semanalmente e discutido nos grupos de pesquisadores intitulados "HumaITas" e outro chamado "CORES", que pertence a Universidade Federal do Rio de Janeiro (UFRJ). Estes grupos são compostos por 
mestres e doutorandos que semanalmente trocam experiências e contribuem uns com os outros para o bom desenvolvimento da pesquisa de modo que todos os protocolos apresentados e ideias são discutidos e colocadas à prova. A coordenação de ambos os grupo está a cargo de professoras doutoras que atuam como pesquisadoras na área de ciência da computação e que participam e conduzem todas as reuniões dos grupos. Com isso, cada fase, pergunta e afins foram diversas vezes questionados e revisados exaustivamente pelo grupo, assim como, os resultados obtidos. Com isso, houve a garantia da qualidade e redução ao máximo possível no viés que poderia ser aplicado. No final, os resultados foram todos revisados pelos grupos e aprovados.

\section{Considerações finais}

O principal objetivo desta RSL foi proporcionar uma visão geral do que tem sido investigado no contexto de recomendações de recrutamento em ecossistemas de startups. Para cumprir este objetivo, seguimos a metodologia de revisão sistemática de literatura descrita por [Kitchenham 2004]. Para tal, concluímos que as dúvidas que tínhamos sobre a área pesquisada foram sanadas e compartilhamos este conhecimento com a comunidade científica através deste trabalho. Infelizmente não foi possível pelo tamanho limite deste, trazer maiores detalhamentos sobre a nossa pesquisa, mas, reservamos um endereço web ${ }^{2}$ com a lista dos 33 estudos observados que acreditamos que contribuirão para futuras outras pesquisas na mesma área que a nossa.

\section{Agradecimentos}

Os autores agradecem o suporte financeiro provido pelo Conselho Nacional de Desenvolvimento Científico e Tecnológico - CNPq (Processo 131162/2020-9).

\section{Referências}

White, H. C. (2013). Chains of opportunity. Harvard University Press.

S. L. Jack D. P. Soetanto. 2013. Business incubators and the networks of technologybased firms. Journal ofTechnology Transfer (2013), 432-453.

Tore Dyba, Torgeir Dingsoyr, and Geir K Hanssen. 2007. Applying systematic reviews to diverse study types: An experience report. (2007), 225-234.

R. K. Ikenami. 2016. A abordagem ecossistema em teoria organizacional: fundamentos e contribuições. Escola Politécnica, Universidade de São Paulo. (2016).

Barbara Kitchenham. 2004. Procedures for performing systematic reviews. Keele, UK, Keele University 33, 2004 (2004), 1-26.

Birgit Pröll and Hannes Werthner. 2005. Lecture Notes in Computer Science.

Claes Wohlin, Per Runeson, Martin Höst, Magnus C Ohlsson, Björn Regnell, and Anders Wesslén. 2012. Experimentation in software engineering. Springer Science \& Business Media.

\footnotetext{
${ }^{2}$ Artigos selecionados:

https://github.com/Leomhl/masters degree archives/blob/master/rsl/artigos selecionados.md
} 